\title{
Diameter Control for Silicon Rod Growth
}

\author{
Rihards Fuksis ${ }^{1}$, Mihails Pudzs ${ }^{1}$, Alexey Kravtsov ${ }^{2}$, Anatoly Kravtsov ${ }^{2}$ \\ ${ }^{1}$ Institute of Electronics and Computer Science, Riga, Latvia \\ ${ }^{2}$ SIA “KEPP EU”, Riga, Latvia
}

Keywords: Silicon rod growth, image processing, meniscus radius measurement.

\begin{abstract}
In this paper we describe a system for diameter control of silicon rod growth by using PID control and image processing algorithms. Image processing algorithms are developed for measuring the radius of meniscus of silicon rods while pulling from the melt. We describe the image processing steps that are performed for segmentation of meniscus region line filtering in complex domain and radius estimation that is based on the bisectors of chords algorithm by incorporating angular information. We test 3 radius measurement methods of meniscus region and analyze the stability on synthetic images as well as on video from the growth process. We also performed an experiment where silicon rod was grown using the developed image processing and control algorithm. We obtained the final result of $2 \mathrm{~mm}$ radius stability on the silicon rod.
\end{abstract}

\section{Introduction}

Silicon crystals, grown using the Float zone (FZ) method have low content of defects, and can be used in the manufacture of crystalline silicon solar cells with high efficiency. However, conventional production of silicon rods - raw material for the FZ process - using slow vapour deposition in the Siemens process has significantly higher cost than production of raw materials for other crystal growth methods. Previously, we have demonstrated a new process for competitive production of silicon rods [1], schematically outlined in the Fig. 1. Growing of the rod is performed by dipping a seed crystal into the melt and then slowly pulling upwards while rotating. The raw material is melted and then heated using electron beams emitted from two electron guns. For each electron beam, focal spots where the heat is transferred to the melt move along the trajectory of half-circle arc with specified radius. Radii of focal spot movement trajectories, as long as the amount of the transferred power form the temperature conditions of the process. At the current stage of our research, we hold these parameters constant during the rod growth process.

One of the problems encountered during early growth experiments was the substantial variation of the diameter of grown rods [1]. Since the goal is to grow a cylindrical rod with stated diameter, the aim of our present work is to enhance the process and introduce the control mechanism for diameter stabilization. In this work we describe the rod growing mechanism, algorithm of the control mechanism and present the acquired results. 


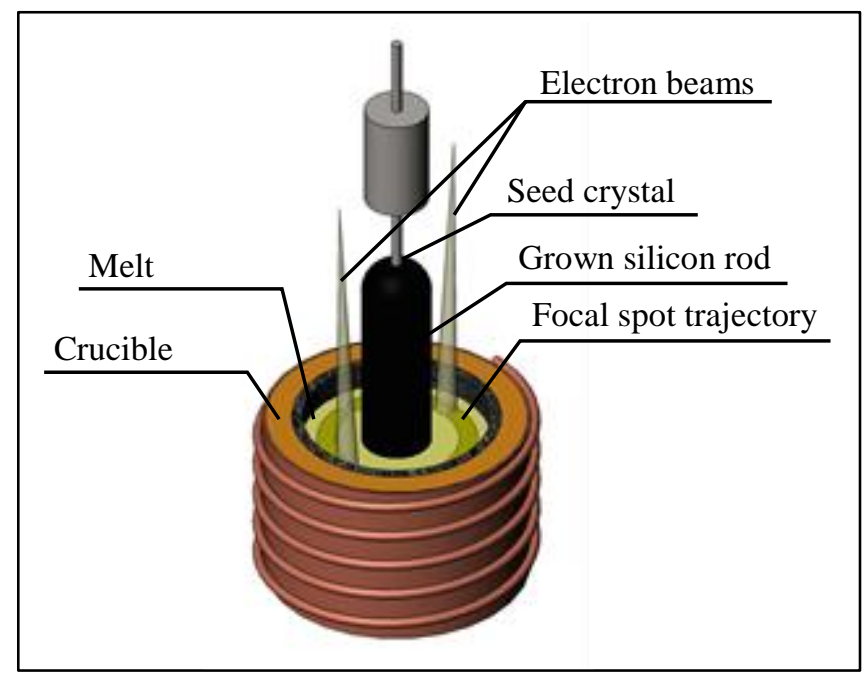

Fig. 1: An outline of the rod growing process

\section{Automatic control of the growth}

Similarly as in the well-known Czochralski crystal growth method, we employ an optical closedloop control method. For this reason, the device is equipped with a video camera that provides a video representation of the process. We measure the diameter of bright arc around the growing rod (Fig. 3, left). This arc is an optical phenomenon formed by reflection of the light from the meniscus. Measured diameter of the meniscus serves as an estimate of the diameter of grown rod and is used as the input variable for the control system. When the focal spot movement trajectories and the power transferred to the melt are constant, the factor that influence the diameter of grown rod is its pulling speed. Change in the pulling speed is the output variable of the control system. Figure 2 outlines the implemented control mechanism.

PID controller takes as an input the deviation value between radius of meniscus $R$ determined by the image processing algorithm and reference radius $R_{r e f}$. PID controller manipulates the reference value $v_{p}$ ref of the pulling speed, by an amount $\Delta v_{p}$. This value indicates a deviation of the real pulling speed trajectory from its pre-calculated reference value. Employing this strategy, system can react to perturbations occurring in crystal diameter.

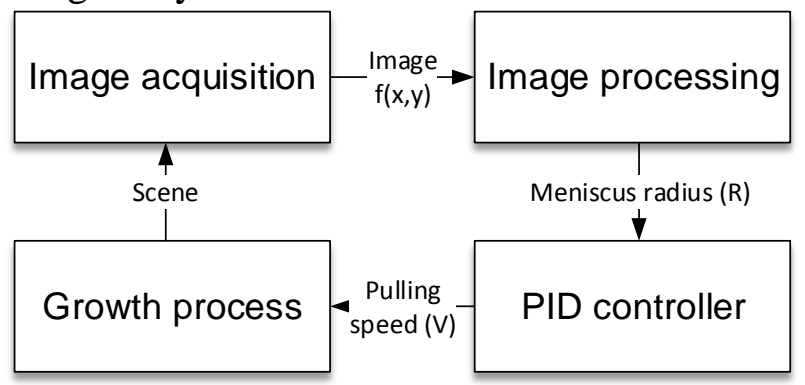

Fig. 2: Block diagram of the control system

Further in this paper we will mostly concentrate on the image processing algorithm analysis and not get in the depth about PID controllers and their usage.

\section{Image processing}

Before radius measurements on the meniscus can be performed, it is necessary to extract the meniscus region from the image. Growth method, where the melt is heated by using two electron beams that run on a circular trajectory, rises additional challenges to measure the radius of meniscus. Because of the bright spots left behind the places of electron collision with the melt, we first apply several enhancement and segmentation steps. The overall workflow of the image processing 
algorithm can be divided in following steps: perspective correction, segmentation and radius measurement. Each of these steps will be described in following subsections.

\section{Perspective correction}

The camera that captures images of the growing process is mounted above the crucible, so it sees a meniscus region and rod from the above at a slight angle. It should be considered that the level of melt decreases during the process. Therefore, the meniscus ring is observed only partially and in perspective under the non-constant angle (e.g. Fig. 3).
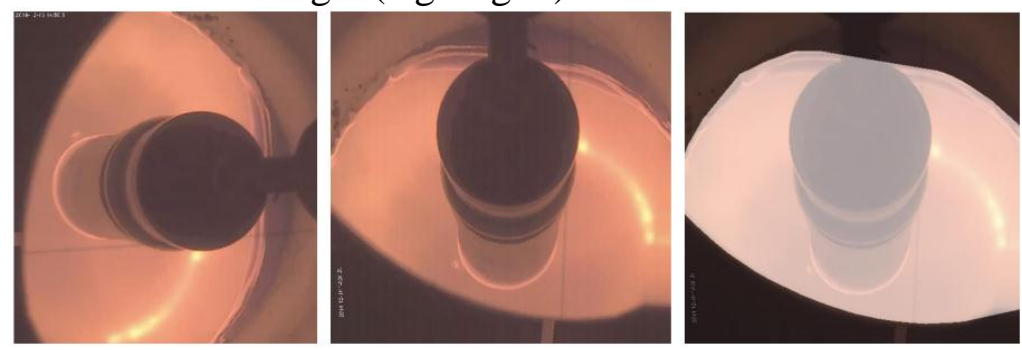

Fig. 3. Input image; image after perspective correction; and extracted ROI

We use affine transformations on the input image to correct the perspective distortions. Because camera is fixed during the growth procedure, we use a checkerboard pattern to obtain calibration matrix before the growing process has started. After corners of the square checkerboard are detected, the coordinate transformation matrix can be calculated. This matrix transforms coordinates from within the plane of the calibration checkerboard to equivalent screen coordinates and can be used to remap pixels of the input image. During the growth process, pixels of every frame are mapped using this transformation matrix before processing. Thus, we ensure that the processed meniscus shape is as close to a circular arc as possible.

\section{Segmentation}

The segmentation is performed to select only the pixels that belong to the meniscus and is crucial for some of the circle detection methods. Meniscus region of interest (ROI) is obtained by following steps shown in block diagram in Fig. 4.

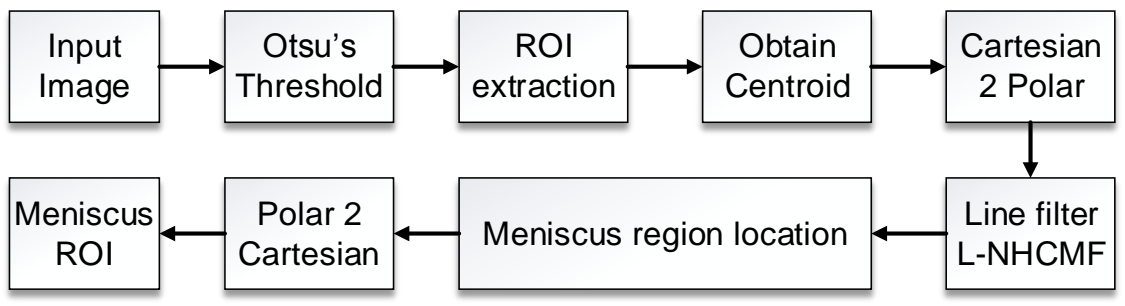

Fig. 4. Block diagram of ROI extraction algorithm

After the input image is obtained from the camera, we need to select the main ROI in which all of the image processing will be performed. For this task, we first perform clustering-based image thresholding by using Otsu's method. After thresholding, the largest non-zero region that represents the melt area is selected. To include in the ROI also the part of the melt covered by the rod, we perform convex hull operation on the obtained region. The resulting ROI includes melt, meniscus and rod. The input image from the camera during the silicon growth process, image after perspective correction and obtained main ROI are shown in the Fig. 3, right. Next, we find the centroid of the main ROI and use it as a center for conversion of the image into polar coordinate system. Since calculated centroid is guaranteed to be close to the middle of the circular meniscus ring, the polar coordinate system is a natural choice for detection of meniscus ROI which is of a circular shape.

\section{Filtering in complex domain}

After converting the image into polar coordinate system, we apply line extraction filter. As we show later, we are also interested in acquisition of additional information about the extracted lines, i.e. orientation. Several approaches can be used for this purpose. For example, Hessian matrix allows to extract line-like objects with the specified brightness (compared to the background) and width, and 
to estimate their direction. However, the line detection is accomplished using modified version of the Non-Halo Complex Matched Filter (NH-CMF) [2].

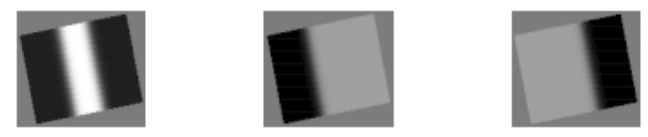

Fig. 5: Left: Bright line; middle and right: left and right edge detection kernels

Originally, NH-CMF is applied by rotating the line detection kernel $M(x, y)$, shown in Fig. 5, left, $\left(\varphi_{n}=\frac{n \cdot \pi}{N}\right)$ and convolving it with the input image $f(x, y)$. Thus, in each pixel $N$ filtering subresponses $s_{n}(x, y)$ are acquired:

$$
s_{n}(x, y)=f(x, y) \otimes M\left(x, y ; \varphi_{n}\right),
$$

The filtering is followed by removal of Halo artifacts and combination of sub-responses into a single vectorial response $\vec{v}(x, y)$ :

$$
\begin{gathered}
\vec{c}(x, y)=\sum_{n=0}^{N-1} R\left[s_{n}(x, y)\right] \cdot e^{2 i \cdot \varphi_{n}}, \\
\vec{v}(x, y)=|\vec{c}(x, y)| \cdot e^{0.5 i \cdot \operatorname{Arg}[\vec{c}(x, y)]},
\end{gathered}
$$

where $R[x] \equiv 0.5 \cdot(|x|+x)$ is the Ramp function. To avoid extraction of the object edges we modify the first step of the original algorithm. Instead of filtering image with line detection kernel (Fig. 5, left), we filter the image with two edge detection kernels $M_{L}(x, y)$ and $M_{R}(x, y)$, shown in Fig. 5 middle and right. When processed image fragment contains an edge, only one kernel will respond with the positive value; on the contrary, in the presence of a line - both kernels respond positively. Assuming that kernel sub-responses are denoted as $s_{L, n}$ and $s_{R, n}$, in the modified algorithm the total sub-response is calculated as:

$$
s_{n}(x, y)=2 \cdot\left(s_{L, n}(x, y)+s_{R, n}(x, y)\right)-R\left[s_{L, n}(x, y)\right]-R\left[s_{R, n}(x, y)\right]
$$

Further processing steps are identical to 2 and 3 (c.f. expressions (2) and (3)).

\section{Meniscus ROI estimation}

Next we search for the brightest points in every column and then classify them by intensity and distance from the polar coordinate center. Points representing meniscus region are the brightest and nearest pixels to the center of polar coordinate system. Image converted to polar coordinate system, image filtered with L-NH-CMF and obtained meniscus ROI are shown in Fig. 6. After obtaining points representing meniscus region we convert them back to Cartesian coordinate system and by performing morphological dilation we slightly enlarge the meniscus region.
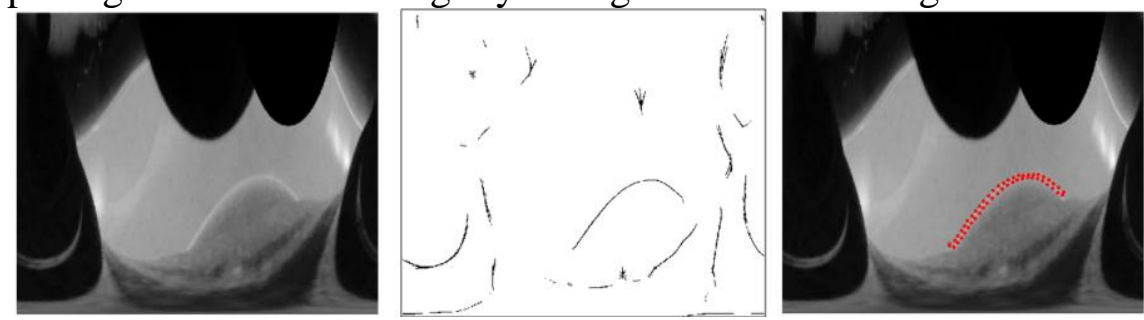

Fig. 6: Left: Image in polar coordinate system; middle: image after L-NH-CMF; right: obtained meniscus region

After we have obtained the meniscus region, we apply circle detection and radius measurement algorithms on all non-zero pixels within meniscus region.

\section{Radius measurement}

Circle detection includes the determination of its center and radius. There are several circle detection methods presented previously, however, not every method can be used when dealing with a half circle or an arc, as in the case of meniscus of the growing rod. Most widely used method for circle detection is Hough Transform (HT), introduced in [3]. HT is a raster voting approach, which 
means that during the analysis of the image, possible candidates for the circle centers are accumulated in 3D spatial array of votes, called accumulator. Although many modifications of HT exist [4], [5], which can reduce the size of the required accumulator memory, the performance of the method is still dependent upon the size of the chosen accumulator. Less complex circle detection methods are vectorized, i.e. work with geometrical primitives, such as points and lines, to calculate and accumulate votes. They are also more sensitive to the distortions of the input data - it must be provided that most of the processed edges come from the same circle. In our setup, this is ensured by the segmentation procedure.

A few simple and effective vectorized circle detection methods were introduced by Li-qin et.al. edge normals [6] and perpendicular bisector of chords [7].

First method relies on the fact that two normals to the circle edges intersect in the center of the circle. Given $N$ edge points, a total of $N \cdot(N-1) / 2$ pairs of edge points can be analyzed. However, to reduce the complexity of algorithm, fewer pairs of edge points can be selected at random. For each analyzed pair of edge points $\left\{\left(A_{x}, A_{y}\right),\left(B_{x}, B_{y}\right)\right\}_{i}$, center candidate $(a, b)_{i}$ can be calculated as follows:

$$
\left\{\begin{array}{l}
a=A_{x}-\frac{\overrightarrow{\boldsymbol{A}}_{x} \cdot\left(\overrightarrow{\boldsymbol{B}}_{y} \cdot\left(A_{x}-B_{x}\right)-\overrightarrow{\boldsymbol{B}}_{x} \cdot\left(A_{y}-B_{y}\right)\right)}{\overrightarrow{\boldsymbol{A}}_{x} \cdot \overrightarrow{\boldsymbol{B}}_{y}-\overrightarrow{\boldsymbol{A}}_{y} \overrightarrow{\mathbf{B}}_{x}} \\
b=A_{y}-\frac{\overrightarrow{\boldsymbol{A}}_{y} \cdot\left(\overrightarrow{\boldsymbol{B}}_{y} \cdot\left(A_{x}-B_{x}\right)-\overrightarrow{\boldsymbol{B}}_{x} \cdot\left(A_{y}-B_{y}\right)\right)^{\prime}}{\overrightarrow{\boldsymbol{A}}_{x} \cdot \overrightarrow{\boldsymbol{B}}_{y}-\overrightarrow{\boldsymbol{A}}_{y} \overrightarrow{\boldsymbol{B}}_{x}}
\end{array}\right.
$$

where $\overrightarrow{\boldsymbol{A}} \equiv\left(\overrightarrow{\boldsymbol{A}}_{x}, \overrightarrow{\boldsymbol{A}}_{y}\right)$ and $\overrightarrow{\boldsymbol{B}} \equiv\left(\overrightarrow{\boldsymbol{B}}_{x}, \overrightarrow{\boldsymbol{B}}_{y}\right)$ are unit normal vectors for edges in points $A$ and $B$ (c.f. Fig. 7, left). Radius candidate $r_{i}$ is then estimated as the mean distance between edge points $A_{i}, B_{i}$ and the center candidate $(a, b)_{i}$. Circle center and radius are obtained statistically from an array of candidate values.
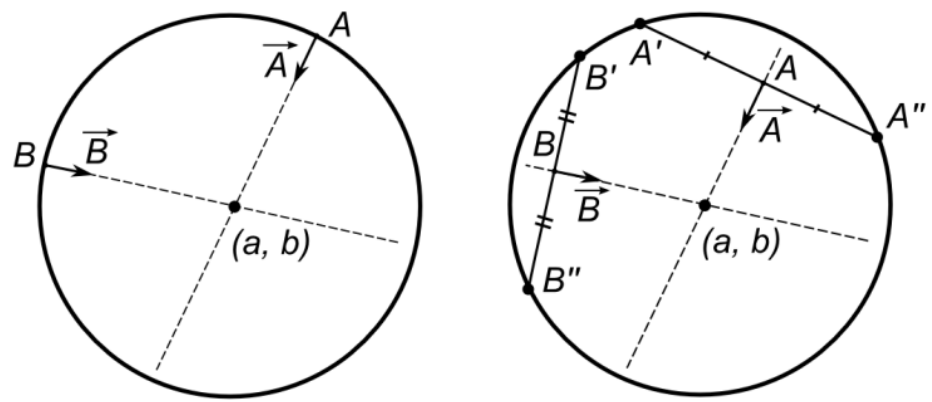

Fig. 7: Illustration for edge normal (left) and bisector of chords (right) methods

Despite the simplicity of this method, its performance is limited by the algorithm for estimation of edge direction. Imprecise edge detection results in the skewed normals and incorrect intersection points (center and radii candidates). The most important factors that influence the precision of center estimation are:

1. difference of edge angles $(\operatorname{Arg}[\overrightarrow{\boldsymbol{A}}]-\operatorname{Arg}[\overrightarrow{\boldsymbol{B}}])$ (perpendicular edges provide the most precise center estimation),

2. angles $\operatorname{Arg}[\overrightarrow{\boldsymbol{A}}]$ and $\operatorname{Arg}[\overrightarrow{\boldsymbol{B}}]$ (it can be shown that the propagated angular error can be significantly larger than the propagated coordinate error).

Other approach, called perpendicular bisector of chords [7] improves the observed drawback. This method uses pairs of perpendicular bisectors of each two chords in the circle. For example, in Fig. 7, right, two chords $A^{\prime} A^{\prime \prime}$ and $B^{\prime} B^{\prime \prime}$ and their respective perpendicular bisectors are shown - it can be seen that these bisectors intersect at the center of the circle $(a, b)$. Given $N$ edge points, a total of $\frac{3 \cdot N !}{(N-4) ! \cdot 4 !}$ combinations of chords can be analyzed, which is significantly more than for the previous approach. However, similarly as in the previous example, we can randomly select fewer quadruples of points $\left\{A^{\prime}, A^{\prime \prime}, B^{\prime}, B^{\prime \prime}\right\}_{i}$ and for each group to calculate the center candidate (intersection point of bisectors) $(a, b)_{i}$, and radius candidate (mean distance from edge points 
$A^{\prime}, A^{\prime \prime}, B^{\prime}, B^{\prime \prime}$ to the center candidate $\left.(a, b)\right)$. To analyze the precision of this algorithm we consider following argument: suppose a chord is given by two pairs of coordinates, i.e. $\left(A_{x}^{\prime}, A_{y}^{\prime}\right)$ and $\left(A_{x}^{\prime \prime}, A_{y}^{\prime \prime}\right)$. Then, the coordinates of the chord midpoint can be calculated as: $A_{x}=\frac{\left(A_{x}^{\prime}+A_{x}^{\prime \prime}\right)}{2}$ and: $A_{y}=\frac{\left(A_{y}^{\prime}+A_{y}^{\prime \prime}\right)}{2}$. Provided equal standard deviation $\sigma^{\prime}$ for the detected endpoints and the independence, the error of calculated center is smaller $-\sigma=\frac{\sigma^{\prime}}{\sqrt{2}}$. Moreover, the tangent unit vector of chord bisector can be calculated as:

$$
\overrightarrow{\boldsymbol{A}}_{x}=\frac{A_{y}^{\prime}-A_{y}^{\prime \prime}}{l_{A}} \quad \overrightarrow{\boldsymbol{A}}_{y}=-\frac{A_{x}^{\prime}-A_{x}^{\prime \prime}}{l_{A}}
$$

where $l_{A}$ is the length of the chord $A^{\prime} A^{\prime \prime}$. The partial derivatives method show that the propagated angular error is dependent of the length of the chords, i.e:

$$
\begin{array}{ll}
\frac{\partial \vec{A}_{x}}{\partial A_{x}^{\prime}}=-\frac{\left(A_{y}^{\prime \prime}-A_{y}^{\prime}\right) \cdot\left(A_{x}^{\prime \prime}-A_{x}^{\prime}\right)}{l_{A}^{3}} & \epsilon O\left(\frac{1}{l_{A}}\right) \\
\frac{\partial \vec{A}_{x}}{\partial A_{y}^{\prime}}=\frac{1}{l_{A}}-\frac{\left(A_{y}^{\prime \prime}-A_{y}^{\prime}\right)^{2}}{l_{A}^{3}} & \epsilon O\left(\frac{1}{l_{A}}\right)
\end{array}
$$

and etc. The longer are the observed chords, the more precise are directions of their respective bisectors. With a proper selection of analyzed chords, subpixel precision can be achieved.

We summarize our analysis by mentioning two conditions that are checked during the random analysis of chords:

- length criteria - the chords are analyzed only if their respective lengths are at least 5 pixels. Thus, we ensure that the impact of the coordinate error on the calculated bisectors is suitable;

- angle criteria - the chords are analyzed only if the angle between their appropriate bisectors is in interval of $\left[\frac{\pi}{6}, \frac{5 \pi}{6}\right]$. Thus, we ensure that the influence of bisector angle error is suitable.

Since edges, obtained by L-NH-CMF are multiple pixels in width, in order to increase the precision of the method further, we propose a modified approach for selection of the chord endings. A vector $\vec{v}(x, y)$, obtained by the L-NH-CMF filter, points the direction of extracted edge line. This information might be used effectively: pixel values that are read (using bilinear interpolation) perpendicularly to this vector, form a certain function $f(t)$, which we call as "cross section". An example of typical cross section is given in Fig. 8, left. For any discrete non-zero starting point $(x, y),(x, y \in Z)$, the cross section can be used to estimate the position of detected line more precisely. In our experiments, we found the median approach to be most robust, i.e. line center $t_{\text {center }}$ is calculated from:

$$
\int_{-\infty}^{t_{\text {center }}} f(t) d t=0.5 \cdot \int_{t} f(t) d t
$$

It must be noted, however, that this operation takes additional time to calculate, compared to the standard chord bisector approach, and is prone to systematic errors if image filtering is not accurate enough. Figure 8, right, demonstrates introduced improvement - each positioned vector in this image begins from a non-zero pixel (detected by the filter) and shows the coordinate correction, applied using the proposed method. The dashed line demonstrates real position of the circle edge used to generate this synthetic image. After the coordinate correction, circle parameters are calculated in the same way as in chord bisector method. 

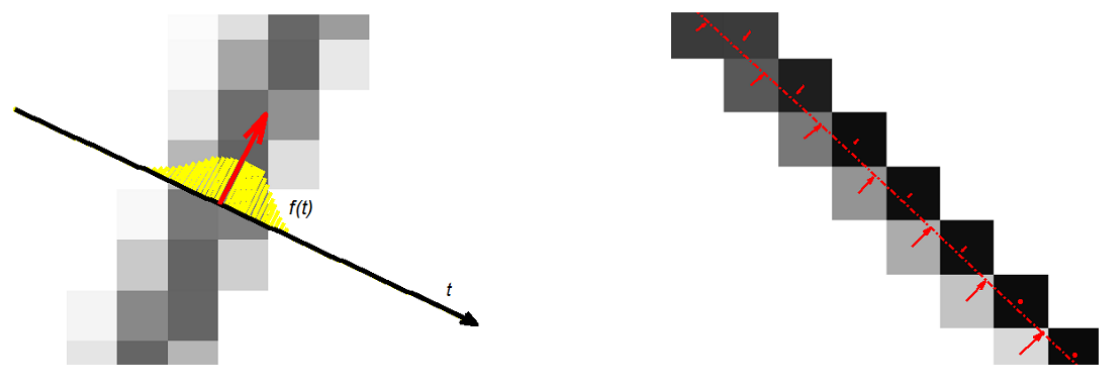

Fig. 8. Left: line and its cross-section values - red arrow shows calculated line center and direction; right: estimation of the line center for each non-zero pixel that is acquired after filtering with L-NH-CMF

\section{Experiments}

From previously described image processing methods, we analyze only radius measurement methods. We do not compare L-NH-CMF filter to other line extraction methods, because other tested methods, such as edge detection and matched filtering, failed to obtain usable segmentation results.

We compare three radius measurement methods: Hough transform, perpendicular bisector of chords, and proposed modified perpendicular bisector of chords that uses vector information obtained from L-NH-CMF. We analyze computation time, precision and accuracy. A quantitative evaluation of the observed approaches is difficult because of the absence of the ground truth data for the meniscus radii. Even though the radius of the grown rod can be thoroughly measured after the process, it doesn't represent the meniscus radius exactly [8]. Therefore, we perform two different experiments. First experiment is carried on a series of synthetic arc images with known radii. Because of the presence of ground truth data, we can evaluate accuracy of the observed circle detection methods. Our second experiment is carried on a series of real growth process images (from a video). The aim of this experiment is to evaluate the consistency of data, i.e. its precision in the real world conditions.

\section{Radius measurement of arcs with various central angles}

It is important that the method used for the radius measurement of meniscus detects radius of a circular arc with central angle of pi or even less. Therefore our first experiment consists of generating synthetic images of circular arcs with various central angles ranging from $[\pi / 4 ; 2 \pi]$ and measuring the radius stability with each of the previously described algorithms. The radius was set to 400 pixels. Results from this experiment are shown in Fig. 9, left. As it can be seen form the Fig. 9 , left, our proposed method is most stable, also when the radius of arc with central angle of $\pi / 4$ has to be detected. It is noticeable that HT tends to converge to $R+1$ pix value. This can be because HT measures the outside part of the arc.
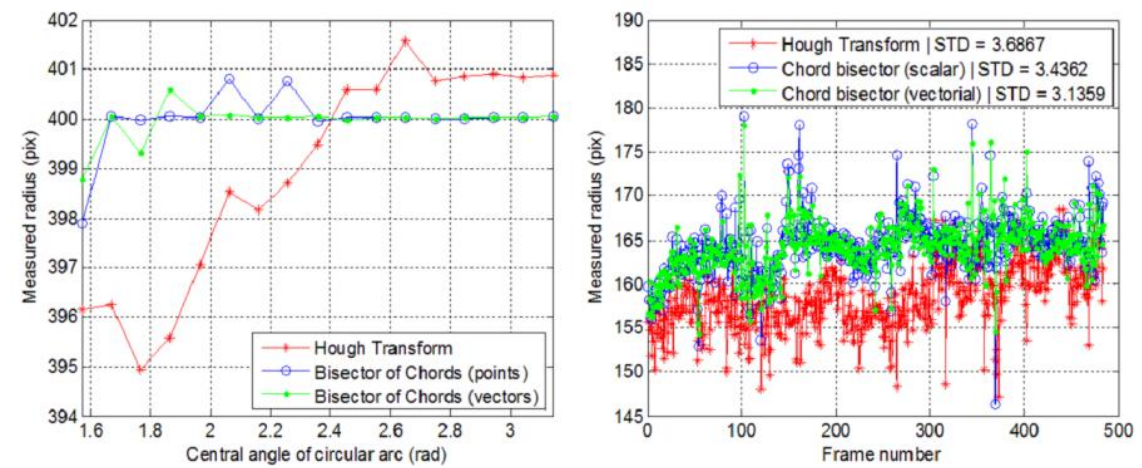

Fig. 9. Left: radius measurement of arcs with different central angles; right: radius measurement of meniscus from the silicon growth video

Radius measurement of meniscus 
We have recorded a video of silicon rod growth and processed 500 frames to evaluate precision of radius measurement. Each circle detection method measures the value $r_{\text {measured }} \equiv r_{\text {true }}+\Delta r$, which differs from the true radius value $r_{\text {true }}$ by some $\Delta r$. If we assume the independence between $r_{\text {true }}$ and error $\Delta r$, the standard deviation:

$$
\operatorname{STD}\left(r_{\text {measured }}\right)=\sqrt{\operatorname{Var}\left(r_{\text {true }}\right)+\operatorname{Var}(\Delta r)}
$$

can be used to compare the precision of each method $\sqrt{\operatorname{Var}(\Delta r)}$, because the value of $\sqrt{\operatorname{Var}\left(r_{\text {true }}\right)}$ is identical for all compared methods. Obtained results are shown on the right side of the Fig. 9. The ratio of processing times for $\mathrm{HT}$, scalar and vectorial bisector of chords is $27: 1: 13$.

In $3.4 \%$ of the frames segmentation fails to extract an arc of at least $\pi / 4$ central angle, therefore, these frames are ommited from the final evaluation. In a system, a sliding buffer of last 30 measured radius values is used to calculate the median value. In this way we eliminate segmentation errors and obtain less varying estimated radius value. For reference, images of rod grown in manual mode and in automatic mode are shown in the Fig. 10.
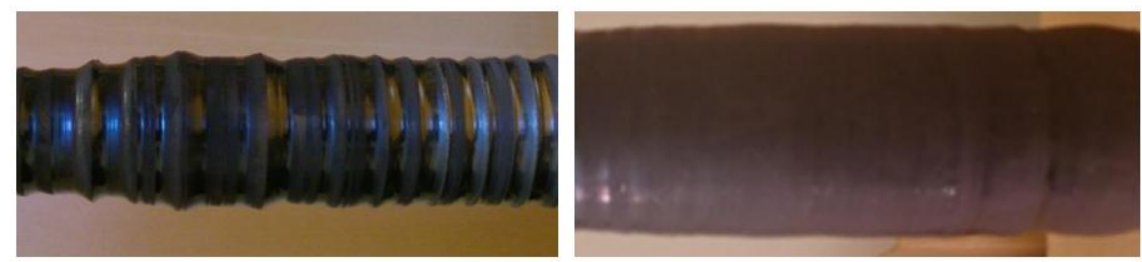

Fig. 10: Left: rod grown in manual mode; right: rod grown in automatic mode

\section{Conclusions}

In this paper we have described algorithms for automatic control of rod growth process. In particular, we first examined the enhancement and segmentation of the input image to obtain the pixels of the meniscus. Next, the circular shape of the meniscus was analyzed using three circle detection methods: raster - HT, and vectorial - original and modified perpendicular bisectors of chords. We compared these methods in terms of accuracy (on synthetic images), precision and processing time (on rod growth video). HT (phase coding implementation) is the slowest approach with least performance, the original bisectors of chords is the fastest approach, but its performance can be further improved by additional analysis of the information of L-NH-CMF vectors, at the expense of the processing time. To guide proper implementation of the vectorial circle detection methods, we also analyzed the conditions that cause degradation of the precision. We provided the visual comparison of the rods, grown in manual mode and in automatic mode, using described approach.

\section{Acknowledgements}

This work was supported by the Project "Competence centre of Latvian electric and optical equipment production" co-financed by the European Union (agreement no. L-KC-11-0006).

\section{References}

[1] Kravtsov A.: Ingots pulled with electron beam heating from skull - a new feed-stock for fz crystals applicable for solar cells. In: Photovoltaic Specialist Conference (PVSC), 2014 IEEE $40^{\text {th }}$. pp. 2991-2993 (June 2014)

[2] Pudzs, M., Greitans, M., Fuksis, R.: Complex $2 d$ matched filtering without halo artifacts. In: Systems, Signals and Image Processing (IWSSIP), 2011 18th International Conference on. pp. 1-4 (June 2011)

[3] Duda, R.O., Hart, P.E.: Use of the Hough transformation to detect lines and curves in pictures. Commun. ACM 15(1), 11-15 (Jan 1972) 
[4] Yuen H.K., Princen J., Illingworth J., Kittler J.: Comparative study of Hough transform methods for circle finding. Image Vision Comput. 8(1), 71-77 (Feb 1990)

[5] Davies E.: A modified Hough scheme for general circle location. Pattern Recognition Letters 7(1), 37-43 (1988)

[6] qin Jia L., zhang Peng C., Liu H.M., Wang Z.H.: A fast randomized circle detection algorithm. In: Image and Signal Processing (CISP), $20114^{\text {th }}$ International Congress om. vol. 2, pp. 820823 (Oct 2011)

[7] Lou L., Xu D., Zhang Z., Zhang J., Qu W.: A fast and robust circle detection method using perpendicular bisector of chords. In: Control and Decision Conference (CCDC), $201325^{\text {th }}$ Chinese. pp 2856-2860 (May 2013)

[8] Winkler, J., N.M.R.J.: A review of the automation of the Czochralski crystal growth process. Acta Physica Polonica A 124(2), 181-192 (August 2013) 